\section{P.3.24 PREVALENCE OF NON-FATAL INJURIES IN INFORMAL WASTE RECYCLERS IN LANDFILL SITES IN JOHANNESBURG, SOUTH AFRICA}

\begin{abstract}
${ }^{1,2,3}$ Nisha Naicker* ${ }^{*}{ }^{1}$ Tahira Kootbodien, ${ }^{1}$ Vusi Ntlebi, ${ }^{1,3}$ Kerry Wilson, ${ }^{1}$ Felix Made, ${ }^{1}$ Nonhlanhla Tlotleng, ${ }^{1}$ Mpume Ndaba, ${ }^{1,3}$ Spo Kgalamono, ${ }^{1,2,3}$ Angela Mathee. ${ }^{1} \mathrm{NIOH}$, Johannesburg, South Africa; ${ }^{2}$ University of Johannesburg, Johannesburg, South Africa; ${ }^{3}$ University of the Witwatersrand, Johannesburg, South Africa; ${ }^{4}$ South African Medical Research Council, Johannesburg, South Africa
\end{abstract}

\subsection{6/OEM-2019-EPI.282}

Background Waste recyclers at landfill sites separate, collect and sell recyclable solid waste. They do not receive Occupational Health and Saftey (OHS) training, personal protective equipment (PPE) or health support. There is limited information of the extent of adverse health outcomes experienced by waste recyclers in South Africa. The objective of this study was to assess the prevalence and possible risk factors for injuries experienced by waste recyclers.

Methods A cross sectional study was conducted among waste recyclers working in two landfill sites. Interviews were conducted with 361 waste recyclers using a structured questionnaire with information on socio-demographic history, exposures and injuries experienced. Logistic regression with OR was used to determine the significant risk factors for having experienced an injury in the last 6 months.

Results The average age was 33.7 years, range of 18-81 year. The majority were male (73.4\%) and had completed secondary school education $(77.8 \%)$. The average income earned was $\$ 119$ per month and 51\% lived in informal dwellings. Exposures included rodents (91\%), lifting heavy object (87\%), dogs (78\%), chemicals (66\%), needles (43.\%), falling waste $(38 \%)$ and violence (20\%). Although $90 \%$ of participants stated they wore some kind of PPE, this was not always suitable for the work conducted. 33\% experienced an injury. The commonest injuries were bitten by rats (90\%), falls on site (46.7\%), muscular pain (29\%), dog attacks (21\%), violence from other waste pickers (20\%) and needle stick injuries (20\%). Logistic regression results showed that being male (crude OR 2.94, 95\% CI- 1.399-6.195) and age <34 years (crude $\mathrm{OR}=2.69,95 \% \mathrm{CI}$ 4.145-4.988) were significant risk factors for injuries.

Conclusion There is a high prevalence of injuries experienced by waste recyclers at landfill sites. OHS awareness and use of appropriate PPE should be provided to waste recyclers in order to prevent occupationally related injuries.

\section{P.3.25 LAPAROSCOPIC SIMULATION FOR VETERINARY STUDENTS}

Riin Raimla*, Marin Loorits, Eda Merisalu. Estonian University Of Life Sciences, Tartu, Estonia

\subsection{6/OEM-2019-EPI.283}

Several laparoscopic simulations have constructed for students to practice skills and precise techniques. Simulations are effective technical tools for teaching professional skills. The aim of the work was to construct a laparoscopic simulation prototype, measure the improvement of manual skills among veterinary medicine students and discomfort in different body parts and assess the need for laparoscopic simulation in education. Simulation had two tasks: coordination and focus- and precision exercise. The number of errors and duration of task time have measured and the results showed that the number of errors and time taken to complete the tasks decreased. In the coordination exercise the total scores improved significantly in the third $(p<0,01)$, fourth and fifth $(\mathrm{p}<0,001)$ performances, compared to the first performance. In the focus- and precision exercise the total scores of the second $(\mathrm{p}<0,05)$ and the third performances $(p<0,01)$ improved significantly, compared to the first performance. In general, strain in different body regions was slightly increased, but no any differences have observed during the task performance. The simulation considered as an important learning tool to improve the manual skills among veterinary students before the practicing in the animal patients.

\section{P.3.27 IMMIGRATION-RELATED STRESSORS AND HEALTH OUTCOMES AMONG LOW-WAGE IMMIGRANT HOTEL WORKERS}

Marie-Anne Rosemberg*. University of Michigan, School of Nursing, Ann Arbor, USA

\subsection{6/OEM-2019-EPI.284}

Background Immigrants (or foreign born individuals) make up about $17 \%$ of US workers and yet over $40 \%$ of the low-wage workforce. Immigrant workers are more likely to be employed in service industries, are paid less, and experience higher rate of injuries and illnesses than their native counterparts. They are exposed to a multitude of hazards in their workplace as well as other stressors outside of work. This study explored stressors leading to ill health among a group of low-wage immigrant workers.

Method We recruited adult immigrants working as hotel housekeepers- a representative group of low-wage service workers. Participants completed survey questionnaires, the SF12 and the Patient Health Questionnaire (PHDQ-2). Descriptive statistics including mean and standard deviation, frequency, and percentage were calculated. Independent t-test were carried out using SPSS 22.0 software.

Results 22 Mexican immigrants and 8 others participated in the study. Among Mexican immigrants, younger age of migration was significantly correlated with more chronic diseases $(r=0.44, p=0.041)$. Compared to other-foreign-countryborn workers, Mexico-born workers were younger when they first came to US $(t=2.08, p=0.048)$ and experienced less discrimination $(t=3.73, p=0.001)$. The two groups did not differ in other stressors including length of stay, demands of immigration, and money sent to others (Ps>0.05). Mexicoborn workers had less chronic diseases and better SF-12 physical health than the other immigrant workers $(t=2.46$, $\mathrm{p}=0.038 ; \mathrm{t}=2.18, \mathrm{p}=0.037)$. Across both groups, higher demands of immigration were significantly associated with more chronic diseases $(r=0.43, p=0.047)$, poorer SF-12 mental health $(\mathrm{r}=-0.56, \mathrm{p}=0.007)$, and more severe depressive symptoms $(r=0.58, p=0.009)$. Higher everyday discrimination was significantly associated with poorer mental health $(\mathrm{r}=-0.47, \mathrm{p}=0.023)$ and more severe depressive symptoms $(\mathrm{r}=0.66, \mathrm{p}=0.001)$.

Implications Immigrant-specific factors affect Hotel housekeepers' health. Studies are needed to identify the protective factors (e.g. social support, enclaves) that can buffer the stressors affecting the health of these immigrant workers. 